W. BLUMENBERG

\title{
EINIGE BRIEFE ROSA LUXEMBURGS
}

Nachdem bereits früher aus den Archiven des IISG. einige Briefe Rosa Luxemburgs und andere die Tätigkeit der Verfasserin in der deutschen Sozialdemokratie betreffende Dokumente veröffentlicht werden konnten ${ }^{1}$, stellen die hier mitgeteilten Briefe eine kleine Nachlese an Luxemburg-Dokumenten dar. Es handelt sich um einen Brief an Minna Kautsky, drei Briefe an Luise Kautsky, ein Schreiben an Hans Kautsky und vier Briefe an Fanny Jezierska. Einer der letzteren enthält die Abschrift eines Schreibens Karl Liebknechts an Fanny Jezierska. Es wurde ferner ein Brief Leo Jogiches' hinzugefügt, weil er für Rosa Luxemburg bestimmte Mitteilungen enthält, die der damals im Berliner "Weibergefängnis" Inhaftierten überbracht wurden.

Drei Briefe stammen aus der Vorkriegszeit, der an Minna Kautsky aus dem Jahre I900, dem Beginn der Freundschaft mit der Familie Kautsky; die anderen sind Grüsse aus den im Süden verlebten Ferien. Die übrigen Briefe wurden während des Krieges, in den Jahren I9Is bis 1918 geschrieben. Die Briefe Nr. I und ro wurden im Konvolut der Briefe Rosa Luxemburgs an die Kautskys, die Briefe Nr. 2, 3 und 9 in Luise Kautskys Privatkorrespondenz gefunden; sie blieben alle unveröffentlicht. Die an Fanny Jezierska gerichteten Schreiben kamen I938 aus dem Besitz der Adressatin in den des Instituts.

In den Briefen aus der Kriegszeit wird in verdeckter Form von der politischen Arbeit des Führungskreises der später in der SpartakusGruppe organisierten Linksopposition innerhalb der Sozialdemokratie gesprochen, Jogiches', Liebknechts, Luxemburgs, Mehrings u.a. Das Erscheinen der ersten Nummer der Zeitschrift Die Internationale wird angedeutet; auf die Entstehung der "Leitsätze" der Linksopposition wird hingewiesen. Die Briefe bringen sachlich nicht viel

1 "Einige Briefe Rosa Luxemburgs und andere Dokumente" in: Bulletin of the IISH., Leiden I952, S. 9-39. 
Neues, aber sie zeigen, in welchen Formen das politische Leben dieses Kreises sich abspielte. Allerdings konnte Rosa Luxemburg im Gefängnis die Junius-Broschüre und ihre Aufsätze für die SpartakusBriefe schreiben, und konnte Karl Liebknecht in der Zeit, da er als Armierungssoldat an der Ostfront war, politische Manuskripte, die er in den Nachtstunden verfasste, nach Berlin senden und in der Gefangenschaft ebenfalls seine Aufsätze schreiben; aber diese Tätigkeit war auch zu jener Zeit keineswegs ungefährlich, und sie wurde nur dadurch ermöglicht, dass es immer wieder Helfer gab, die aus Sympathie mit den Gefangenen und ihren Bestrebungen den Verkehr mit der Aussenwelt vermittelten. Immerhin musste bei wichtigeren Mitteilungen besondere Vorsicht beachtet werden. So schrieb Rosa Luxemburg den Brief Nr. 5 aus dem Gefängnis in der Barnimstrasse auf den Rand von acht Seiten einer alten Ausgabe der Werke von Nicolas Boileau-Despréaux. Die Schrift ist bräunlich. Vermutlich wurde der Brief mit Milch geschrieben; sie nimmt, über einer Flamme erhitzt, diese Färbung an, und das starke Papier eignete sich vorzüglich zu dieser Prozedur.

Es ist nicht bekannt, was Luise Kautsky veranlasste, den grossen Brief aus Wronke vom I 5 . April 1917 nicht in ihre Briefsammlungen 1 aufzunehmen. Es ist nicht anzunehmen, dass die begeisterte Begrüssung der russischen Februarrevolution oder der freundschaftlich zurechtweisende und aufmunternde Appell an die Adressatin oder gar die Kritik an Karl Kautsky, - in diesem Fall eines ,bequemen fatalistischen Optimismus, ... der die eigene Impotenz verschleiern soll" -, die Aufnahme verhindert haben; denn alledem gab Rosa Luxemburg auch in anderen Briefen an Luise Kautsky Ausdruck. Was aber den menschlichen Gehalt angeht, so steht dieser Brief neben den wertvollsten der anderen Sammlungen. Er zeigt wieder die ausserordentliche Fähigkeit der Briefschreiberin, jeden Augenblick intensiv zu erleben. Immer mit grossen theoretischen und politischen Problemen beschäftigt, oft enttäuscht und fast verzagend, weiss sie doch jeden Tag im alltäglichen Geschehen, in Natur und Geisteswelt genug des Lebenswerten zu entdecken. Sie hat „stets Ursache, um heiter zu sein”, weil sie „nach Honig in jeder Blüte sucht” und nicht die „üble Gewohnheit" hat, darin Gift zu suchen. Die Intensität des Empfindens, Denkens und Handelns unter Verachtung jedes Dogmatismus - wie charakteristisch ist hierfür wieder ihre Beurteilung Ricarda Huchs! - gibt Rosa Luxemburgs Äusserungen ihren bestechenden Reiz.

1 Rosa Luxemburg, Briefe an Karl und Luise Kautsky (1896-1918), Berlin 1923. - Rosa Luxemburg, Briefe an Freunde, nach dem von Luise Kautsky fertiggestellten Manuskript hrsg. v. Benedikt Kautsky, Hamburg 1950. 
Den Brief Nr. ro wird Luise Kautsky von der Veröffentlichung ausgeschlossen haben, weil er Angaben über das Testament Hans Diefenbachs enthält, der am 25. Oktober 1917 in Frankreich gefallen war, und Missverständnisse über Rosa Luxemburgs finanzielle Verhältnisse erwecken konnte. Tatsächlich kam auch bei Luise Kautsky ein solches Missverständnis auf; denn sie hatte von den Testamentsvollstreckern eine Abschrift des Testaments erbeten und erhalten. Das war möglich, weil sie selbst als Freundin Hans Diefenbachs die Verfügung über einen Betrag erhalten sollte, den dieser testamentarisch zur Bekämpfung des Antisemitismus bestimmt hatte. ${ }^{1} \mathrm{Da}$ dieser Brief biographischen Wert hat, meinten wir jedoch, ihn veröffentlichen zu sollen.

Schliesslich folgt eine „Erklärung” Rosa Luxemburgs aus dem Jahre I90I. Auf dem Lübecker Parteitag der Sozialdemokratischen Partei Deutschlands war von mehreren Rednern die Presse heftig kritisiert worden. In der Hauptsache richtete sich die Kritik gegen die Neue Zeit, insbesondere gegen Parvus und Rosa Luxemburg wegen des aggressiven Tones ihrer Arbeiten über die Bernstein-Debatte, die Millerand-Frage und die Budgetbewilligung in süddeutschen Staaten, sowie gegen Kautsky, der als Redakteur der Zeitschrift die Arbeiten aufnahm. Richard Fischers Ausführungen erhielten dadurch ein besonderes Gewicht, dass er Reichstagsabgeordneter und Geschäftsführer des Vorwärts-Verlages war. Der bissige Schluss der „Erklärung” wird dadurch verständlich, dass Fischer die rassische Abkunft Rosa Luxemburgs erwähnte, eine Bemerkung, die aus seinem Munde und in diesem Zusammenhang nicht eine sachliche Feststellung war. Es gelang Kautsky, Rosa Luxemburg zum Verzicht auf die Veröffentlichung der „Erklärung" in der Neuen Zeit zu bewegen. Aber sie erklärte sich dazu bereit in einem Briefe, ${ }^{2}$ in dem sie bedauerte, dass Kautsky ihr nicht, wie es Freundespflicht gewesen wäre, zur Wahrung ihrer schriftstellerischen Ehre geraten habe: „Der Freund liess sich

1 In einem Schreiben der Rechtsanwälte Diefenbach \& Dr. Schill, Sturtgart, 22, Mai I918, an Luise Kautsky hiess es: „In der Nachlassache Diefenbach habe ich vom Inhalt Ihrer gefl. Zuschrift vom r6. d. Mts. meiner Kusine, Frau Margarete Müller, hier, als Alleinerbin Kenntnis gegeben und übersende Ihnen in der Anlage in deren Einverständnis und in Entsprechung Ihres Wunsches eine Gesamtabschrift des Testaments, bei welcher lediglich die Sie ja weiter nicht interessierenden Beträge weggelassen sind. Ich bemerke noch, dass der von Hans Diefenbach für die Erbschaftssteuer vorgesehene Betrag bei weitem nicht ausgereicht hat, so dass tatsächlich für eine Verwendung zur Bekämpfung des Antisemitismus nichts übrig ist. $\mathrm{Da}$ jedoch Frau Müller in jeder Richtung peinlichst die Wünsche ihres verstorbenen Bruders berïcksichtigen will, so hat sie sich entschlossen, trotzdem einen Betrag hierfür zur Verfügung zu stellen, worüber Ihnen noch nähere Mitteilung zugehen wird..."

2 an Karl Kautsky vom 3. Oktober r9or, s. „Briefe an Karl und Luise Kautsky” S. s6f. 
aber ganz vom Redakteur der Neuen Zeit beherrschen, und dieser will seit dem Parteitag überhaupt nur Eins: er will seine $R$ uhe haben, er will zeigen, dass die Neue Zeit nach den erhaltenen Prügeln artig geworden ist und Maul hält ..."

\section{AN MINNA KAUTSKY 196}

Friedenau, 30. Dezember I900. Wielandstrasse 23.

\section{Verehrte und liebe Freundin!}

Nun will ich wenigstens zur Jahrhundertwende den längst gehegten Wunsch meines Herzens befriedigen und Ihnen einige Zeilen schreiben. Vor allem vielen Dank für die lieben Worte, die Sie mir aus Anlass des Todes meines Vaters geschickt haben. Dieser Schlag hatte mich für Monate so niedergeschmettert, dass ich mit den Menschen weder mündlich noch schriftlich verkehren konnte. Ich war innerlich wie abgestorben und so gleichgültig für alles, dass ich nur mechanisch die Tagesobliegenheiten eines „lebendigen Menschen" verrichtete. - Nun bin ich wieder einigermassen auferstanden, Freude an der Arbeit, Lust an menschlicher Gesellschaft, Freundschaft sind für mich wieder zu Wirklichkeiten geworden. Schade, dass Sie so fern sind, es fehlt mir häufig, wenn ich bei Karl und Luise bin, die liebenswürdige Heiterkeit der Grossmama, ihr so innig freundliches Wesen ... Wann kommt Grossmama wieder nach „Bellin”?

Wir verkehren mit der Karlschen Familie viel, obwohl es immerhin nicht mehr so leicht und bequem geht, wie damals, wo man nur um die Ecke zu biegen brauchte; jetzt muss man schon ganze zehn Min[uten] gondeln zueinander, sich also anziehen, den Entschluss fassen usw.

Diesen Weihnachtsabend haben wir nicht zusammen verbringen können, aber am zweiten Feiertag hat sich bei Karls eine unerwartet grosse Gesellschaft eingefunden, und wir amüsierten uns ausgezeichnet. Mir gefiel besonders ein frischer Gast - Herr Vorländer, der NeoKantianer, den Mehring neulich in der N[euen] Z[eit] besprochen hat. Ein neuer Mensch, aus anderem Milieu, dazu selbst liebenswürdig und gescheit, erfrischt ungemein.2

1 Karl Kautskys Mutter, Vetfasserin damals vielgelesener sozialer Romane.

2 Mehring hatte Karl Vorländers Schrift „Kant und der Sozialismus, unter besonderer Berücksichtigung der neuesten theoretischen Bewegung innerhalb des Marxismus", Berlin 1900, in zwei Aufsätzen der Neuen Zeit sehr wohlwollend besprochen, Jahrg. XVIII Bd. 2 S. Iff., 33 ff. Bald änderte sich seine Stellung zu Kant und den Kantianern; über diese Wandlung s. K. Vorländer, „Kant und Marx”, Tübingen I9r r, S. 227ff. Rosa 
In der allerletzten Zeit habe ich mich mit Mehrings angenähert. Beide erweisen mir eine, meines Erachtens ganz unmotivierte, Neigung, - wie mir denn immer die Sympathie anderer als etwas Unerwartetes, als Geschenk erscheint. Frau Mehring spielt sehr schön Klavier und liebt Beethoven und Chopin, starke Berührungspunkte sind also jedenfalls da.

Ich studiere und geniesse jetzt Hebbel, den ich bis jetzt nicht kannte. Herodes und Mariamne haben mir einige glückliche Stunden bereitet. - Ich schreibe so viel von mir! Aber das ist ja nur eine List, um Sie zu verbinden, mir bald, recht bald einige Zeilen über Ihr Leben und Wohlergehen zu schenken. Prosit Neujahr!

Mit herzlichen Grüssen

Ihre erg[ebene] Rosa L.

\section{AN LUISE KAUTSKY}

[Poststempel: Levanto (Genova), 9. Juni r9o9].

Liebe Lulu!

Ich warte und warte auf das Paket Bücher, um Dir dafür danken und zugleich einen ausführlichen Brief schreiben zu können. Die Bücher kommen aber nicht! ${ }^{1}$ Alle Briefe werden mir von Genua hierher nachgesandt, das Paket ist also nicht angelangt oder weiss der Teufel! Bitte, reklamiere Du auf der Friedenauer Post; denn hier an die italienische zu schreiben, ist schade um die Tinte. Ich bin hier in Levanto schon seit zwei Wochen, es ist herrlich hier, ich bleibe aber wohl nicht zu lange hier, da es heiss wird. Jetzt freilich ist ziemlich kühl. Nächstens schreibe ich Dir ausführlich. Viele Küsse einstweilen für alle

Deine R.

Denke Dir, was mir die hiesige Post eingebrockt hat! Mein Bruder aus Warschau ${ }^{2}$ war als Delegierter auf dem med[izinischen] Kongress für Fabrikunfälle in Rom (er ist Fabrikarzt), war dann in Venedig, schrieb immerzu an mich, ich an ihn, und wir konnten uns nicht treffen infolge von Briefverspätungen! Schliesslich ist er abgereist.

Luxemburg beurteilte Vorländer zu dieser Zeit wie Mehring; er sagte in seinem ersten Aufsatz, was ihn veranlasse, die Schrift Vorländers so ausführlich zu behandeln, sei „in erster Reihe die persönlich loyale und sachlich anregende Weise, womit er sein Thema behandelt. Es lohnt sich in jeder Beziehung, mit ihm zu diskutieren..."

1 R. L. arbeitete in dieser Zeit an der „Einführung in die Nationalökonomie”, die sie auch in der Berliner Parteischule vortrug; die bereits vor dem Kriege geschriebenen und im Nachlass vorgefundenen Kapitel des unvollendet gebliebenen Werkes gab Paul Levi, Berlin 1925, heraus.

2 Dr. Joseph Luxemburg; er war mit den Freunden seiner Schwester befreundet und hatte bereits 1906 die Familie Kautsky in Berlin besucht. 
So eine Sau von einer Post ist noch nicht dagewesen. Ich bin untuhig um die Bücher! Wie schade, dass Du Deine Notizen nicht im Brief geschickt hast!

Meine Adresse hier: Levanto, Riviera di Levante, ferma in posta.

\section{AN HANS KAUTSKY 1}

Herrn Professor Hans Kautsky, Hoftheatermaleur[!], Berlin-Halensee, Ringbahnstrasse I I I.

Lieber Hans!

[Poststempel: Clarens, Is. April 1913].

Besten Gruss Ihnen und Luise vom Genfer See. Im „Schloss” konnte ich nicht wohnen; denn es liegt zu ungünstig, gar keine Spaziergänge, als die eine staubige Fahrstrasse, auf der Automobile immerzu sausen. So bin ich wieder in Chailly. Es war hier wundervoller Frühling in voller Blüte, aber seit zwei Tagen haben wir Winter, Schnee und Kälte. Was macht Gretl? ${ }^{2}$ Ich grüsse sie und hoffe, sie bald zu sehen (falls sie nicht wieder ausfliegt). Ich bin bald wieder in Südende.

Ihre R. L.

\section{AN FANNY JEZIERSKA ${ }^{3}$}

Auguste Victoria-Krankenhaus 4 I6. Januar igrs.

Sehr geehrte Gen[ossin] Jezierska!

Vielen Dank für die schönen Blumen und die Bücher. Ich lese sie mit grossem Interesse. Hoffentlich geht es Ihnen recht gut.

Mit den besten Grüssen Ihre R. L.

1 Karl Kautskys Bruder, der auch nach dem Bruch mit Karl Kautsky zu R.L.'s engerem Freundeskreis gehörte.

2 Hans Kautskys Tochter.

3 Fanny Jezierska hatte in jener Zeit Verbindung mit Russen, Polen und vielen Mitgliedern der Spartakus-Gruppe wie Ed. Fuchs, Mathilde Jacob, Leo Jogiches, Karl Liebknecht, Franz Mehring, Bertha Thalheimer u.a. Sie war in den Jahren 1919-20 im westeuropäischen Sekretariat der Komintern in Berlin tätig, 1922-24 in Italien; 1925 - 29 war sie Sekretärin des EKKI in Moskau. Später emigrierte sie nach Frankreich und starb während des Zweiten Weltkrieges in den USA.

4 Nachdem das gegen R.L. am 20. Februar I914 in Frankfurt a. M. wegen Aufforderung der Soldaten zum Ungehorsam ergangene Gefängnisurteil vom Reichsgericht bestätigt war, erhielt sie wegen Krankheit im Januar I9is Strafaufschub bis zum 31. März. Sie brachte einige Wochen im Krankenhaus zu. Am I8. Februar wurde sie in ihrer Wohnung verhaftet und ins „Weibergefängnis” in der Barnimstrasse eingeliefert. 
5. LEO JOGICHES AN MATHILDE JACOB ${ }^{1}$

W[erte] G[enossin]

2. April I9Is.

In dem Schriftstück, das wir heute zusammen verfasst haben, wollen Sie, bitte, einiges ändern, und zwar:

I. Den Satz: „Franziskus wird seine Arbeit bis zum 10. April fix und fertig machen" 2 bitte $q u$ streichen, Statt dessen sagen Sie folgenden Satz:

„Der Mimi ${ }^{3}$ geht es sehr gut, die Wohnung ist in bester Ordnung, nur ist noch das Scbild an der Tür nicht angebracht, aber Onkel Leo hat versprochen, dass am Ende der näcbsten Woche aucb das Schild wieder aufgehängt sein wird."

2. Den Satz mit Maria Stuart, bitte, streichen zu wollen. Statt dessen muss es heissen:

„Das Töchterchen Karls hat sich über das Geschenk gefreut. Sie versteht das Buch ganz gut und hat es in einem Tag zu Ende gelesen."

Alles andere bleibt unverändert. Besonders ist mir an der wörtlichen Wiedergabe des Satzes mit der „Demarkationslinie” gelegen.

Ich habe leider das Bündel Zeitungen auf dem Tisch liegen lassen, werde es aber nächstens abholen.

Mit bestem Gruss

J. Tyszka.

\section{AN FANNY JEZIERSKA}

[Barnimstrasse, etwa Ende Oktober I9I5]

Mein liebes Frl. Jezierska!

Durch ein für mich rätselhaftes Missverständnis ist ein geschlossener Brief, der augenscheinlich an Sie gerichtet war, mir zugestellt worden. $\mathrm{Da}$ ich erst in einigen Wochen vielleicht die Gelegenheit

1 Leo Jogiches (J. Tyszka), seit I89o mit R. L. befreundet; der eigentliche Organisator und spätere Leiter der Spartakus-Gruppe. Es ist mit Sicherheit anzunehmen, dass der Brief an Mathilde Jacob gerichtet war. Sie war R. L.'s Sekretärin, besorgte deren Korrespondenz besonders in der Zeit, die sie im Gefängnis in der Barnimstrasse verbrachte, und konnte am ehesten die Verbindung zu ihr aufrechterhalten.

a Gemeint sind zweifellos Franz Mehrings Beiträge „Unsere Altmeister und die Instanzenpolitik"und die unter dem Titel „Ein plumper Täuschungsversuch” verfasste Besprechung von H. Cunows Schrift "Partei-Zusammenbruch?", die in der Internationale S. 60-70 bzw. S. 77 f. erschienen. Das einzige Heft der von Luxemburg und Mehring herausgegebenen Zeitschrift Die Internationale. Eine Monatsscbrift für Praxis und Tbeorie des Marxismus erschien am I5. April igrs.

3 R. L.'s Katze. Es handelt sich natürlich bei allen Mitteilungen des Briefes um Anspielungen, deren Sinn die Briefempfängerin verstand. 
haben dürfte, ihn wieder hinauszugeben, so bleibt mir nichts anderes übrig, als ihn Ihnen hier wortgetreu abzuschreiben, um Sie so schnell wie möglich von seinem Inhalt in Kenntnis zu setzen. Dem Brief lag ein Manuskr[ipt] auf acht engbeschr[iebenen] Seiten bei, das ein detailliertes Konzept zu einer Abhandlung über Militarismus darstellt. Dieses muss ich hier aufbewahren bis zur Gelegenheit. Noch eins! Ich finde es höchst gefährlich, dass K[arl] diese Schriftstellertätigkeit auf Entfernung entwickelt, ${ }^{1}$ und Sie würden wohl ein gutes Werk tun, wenn Sie ihn davon in passender Form gleich abmahnen würden.

Herzl[ich] Ihre R. L.

Der Brief lautet:

I8. IO. Is.

[Karl Liebknecht an Fanny Jezierska.]

Liebe Fanja!

Heute kam auch Dein Brief vom Io. Io. Danach hast Du meinen Brief vom 6., nicht aber die zwei früheren erhalten. Ich hoffe doch, dass auch die inzwischen eingetroffen sind - schreib sofort, ob Du den Institut-Entwurf ${ }^{2}$ hast usw. Bezeichne ruhig genau. Ich bin sehr kaput - weil ich meine Arbeit trotz all der militärischen „Arbeit” nicht lassen kann und so nicht zur Ruhe komme. Die Russen sind gestern nacht weit zurückgegangen. Auf einige Zeit liegt also unsere Arbeit hinter der Front. Kannst Du mir die erbetene Arbeitshilfe leisten? Ich weiss mir keinen anderen Rat und baue auf Dich. Aber Du bist stark beschäftigt und nicht kräftig. Übertrag Du's einem anderen - bitte; aber ich weiss nichts, als mich an Dich wenden. Dein neues Paket erwarte ich gern. Der Tabak im ersten war - neben den Cognacbohnen - der Glanzpunkt; könnte aber etwas gröber sein, und ganz billig. Qualität spielt gar keine Rolle. Ich lege Dir hier ein neues Stück Manuscript ${ }^{3}$ bei, wie ich gestern eines schickte. Bitte erledige

1 Karl Liebknecht war als Armierungssoldat an der Ostfront.

2 Vielleicht verschrieben statt „Statuten-Entwurf"? Vermutlich handelt es sich um einen Entwurf der „Leitsätze”, die von Rosa Luxemburg im Einvernehmen mit Karl Liebknecht u.a. verfasst, von einer am I. Januar 1916 in Liebknechts Berliner Büro tagenden Vertrauensmännerkonferenz der Linksopposition beschlossen und im Spartakus-Brief vom 3. Februar 1916 veröffentlicht wurden.

3 Bei Fanny Jezierskas Briefen befinden sich 48 Seiten Manuskript von Karl Liebknecht; es sind Aufsätze „Ein Vorspiel zum Weltkrieg”, „Der Ausbruch der Weltkrieges”, "Worte des Wahns" und Notizen. Der Aufsatz "Worte des Wahns" erschien als Leitartikel unter Liebknechts Pseudonym Implacabilis in Nr. 3 der Jugend-Internationale, Zürich vom I. März 1916. Bei dem von R.L. als „detailliertes Konzept einer Abhandlung über Militarismus" bezeichneten wird es sich um ein anderes Manuskript gehandelt haben. 
es irgendwie. Jedenfalls verwahre es gut und sorg auch, dass es nicht verwischt. Vielen, vielen Dank voraus! Es ist , Uhr früh. Noch eine halbe Stunde Schlaf. Leb wohl - Alles Beste

Ich wiederhole das Datum: i 8 . 10. I5.

herzlich K. L.

7. AN FANNY JEZIERSKA

17. März [1916] ${ }^{1}$

Liebe Genossin Jezierska,

Besten Dank für Ihre Zeilen. Es wäre auch für mich am liebsten, wenn wir am Sonntag ruhig ein paar Stunden plaudern könnten, nur geht das leider noch an diesem Sonntag nicht, da ich den ganzen Tag nicht mir gehöre. Aber haben Sie doch die Güte, mich Ende nächster Woche anzuklingeln, ich hoffe, dass wir uns dann wenigstens für den folgenden Sonntag verabreden können. Dank für die Gelegenheit nach W[arschau?]. Ich würde natürlich gern einen Brief mitgeben, aber das dürfte wohl nicht gut gehen? Mündlich hätte ich sonst nichts als Grüsse zu bestellen und das tue ich ohnehin auf lakonischen Postkarten.

Einstweilen mit herzlichen Grüssen Ihre R. L.

\section{AN FANNY JEZIERSKA}

[O. D. Vermutlich Ende März oder April 1916] ${ }^{1}$

\section{Liebe Genossin Jezierska!}

Anbei mit bestem Dank der Brief, den ich mit grossem Interesse gelesen habe, obwohl ich gestehen muss, dass mir dieses originelle Deutsch das Verständnis einigermassen erschwert hat. Auch für die Zeitungen vielen Dank und bitte um mehr! Für die Russen zu schreiben halte ich aus verschiedenen Gründen für inopportun: ich möchte vorerst nur in der deutschen Presse schreiben, Sie werden wohl die Gründe verstehen. Nun möchte ich Sie doch endlich bei mir sehen und schlage Ihnen vor, an einem Werktag Abends zu mir hinauszufahren: am Sonntag macht sich das leider zu schwer, da dieser Tag immer durch etwas Unaufschiebbares belegt ist. Aber die Tage sind ja jetzt schon lang und man kann auch vom Abend etwas haben. Also

1 R. L. war von Ende Januar bis zum 1o. Juli 1916 in Freiheit. Der Brief muss aus diesem Jahre stammen. 
melden Sie sich, bitte, vor allem telephonisch und dann verabreden wir das Weitere!

Inzwischen mit herzl[ichem] Gruss

Ihre R. Luxemburg

\section{AN LUISE KAUTSKY}

Wronke i. P., I5. April I9I7.

Geliebte Lulu!

Dein kurzes Brieflein vor Ostern hat mich durch seinen äusserst gedrückten Ton lebhaft beunruhigt, und ich habe mir gleich vorgenommen, Dir wieder einmal das Köpfchen zu waschen. Sag mal, wie kannst $\mathrm{Du}$ bloss wie eine traurige Zikade Dein Liedlein der Trübsal weiter singen, während aus Russland ein solch heller Lerchenchor herübertönt?! Begreifst Du denn nicht, dass dies unsere eigene Sache ist, die dort siegt und triumphiert, dass es die Weltgeschichte in Person ist, die dort ihre Schlachten schlägt und freudetrunken die Carmagnole tanzt? Muss man denn nicht alle Privatmisere bei solchem Gang der allgemeinen Sache vergessen? Ich weiss, Dich bedrückt, dass ich gerade jetzt nicht frei bin, um die Funken zu sammeln, die dort stieben, um dort und anderswo zu helfen und zu steuern. Sicher, das wäre schön, und Du kannst Dir denken, wie mir in den Gliedern zuckt, und wie mir jede Nachricht von dort wie ein elektrischer Schlag bis in die Fingerspitzen fährt. Aber das Nichtmitmachenkönnen stimmt mich deshalb nicht um ein Jota trüber, und es fällt mir nicht ein, mir durch Stöhnen über das, was ich nicht ändern kann, die Freude am Geschehenden zu verkümmern.

Siehst $\mathrm{Du}$, ich habe gerade aus der Geschichte der letzten Jahre und von da rückschauend aus der ganzen Geschichte gelernt, dass man das Wirken des einzelnen nicht überschätzen soll. Im Grunde genommen wirken und entscheiden die grossen, unsichtbaren, plutonischen Kräfte der 'Tiefe, und alles rückt sich schliesslich zurecht, sozusagen „von selbst." Missverstehe mich nicht: ich rede da nicht etwa einem bequemen fatalistischen Optimismus das Wort, der die eigene Impotenz verschleiern soll, wie er mir gerade bei Deinem verehrten Gatten verhasst ist. Nein, nein, ich bin allzeit auf dem Posten und werde bei der nächsten Möglichkeit wieder dem Weltklavier mit allen zehn Fingern in die Tasten fallen, dass es dröhnt. Nun ich aber nicht durch meine Schuld, sondern durch äusseren Zwang "auf Urlaub" bei der Weltgeschichte bin, so lache ich mir einen Ast, freue mich, wenn's auch ohne mich geht, und glaube felsenfest, dass es gut gehen wird. 
Die Geschichte weiss stets am besten Rat, wo sie sich am hoffnungslosesten in die Sackgasse verlaufen zu haben scheint.

Liebste, wenn man die üble Gewohnheit hat, in jeder Blüte nach einem Tröpflein Gift zu suchen, so findet man, solange man lebt, eine Ursache zum Stöhnen. Nimm aber die Dinge umgekehrt und suche nach Honig in jeder Blüte, so findest Du stets Ursache, um heiter zu sein. Ausserdem glaube mir, die Zeit, die ich - wie auch andere - jetzt hinter Schloss und Riegel verbringe, ist auch nicht verloren. Sie kommt irgendwie in der grossen allgemeinen Rechnung zur Geltung. Ich bin der Meinung, dass man einfach, ohne zu viel Schlauheit und Kopfzerbrechen, so leben soll, wie man es für recht hält, ohne für alles gleich in bar auf die Hand ausgezahlt kriegen zu wollen. Es wird sich schon alles zum Schluss finden. Und wenn nicht - ist mir "ooch schnuppe"; ich freue mich ja auch schon so des Lebens, inspiziere jeden Morgen gründlich den Knospenstand auf allen meinen Sträuchern, besuche jeden Tag ein rotes Marienkäferlein mit zwei schwarzen Pünktchen auf dem Rücken, das ich seit einer Woche auf einem Ast in einem warmen Verband aus Watte trotz Wind und Kälte am Leben erhalte, beobachte die Wolken, wie sie stets neu und immer schöner sind und - fühle mich im ganzen nicht wichtiger als dieses Marienkäferlein und in diesem Gefühl meiner Winzigkeit unaussprechlich glücklich.

Vor allem: die Wolken! Welcher unerschöpfliche Grund des Entzückens für ein Paar Menschenaugen! Gestern, Sonnabend, stand ich nachmittags gegen fünf Uhr gelehnt an meinen Drahtzaun, der das Gärtlein von dem übrigen Hof trennt, liess mir die Sonne auf den Buckel scheinen und blickte nach dem Osten. Dort türmte sich auf blassblauem Himmelsgrund ein grosses Wolkengebilde von zartestem Grau, über das ein leichter Rosaschimmer wie hingehaucht war; das zauberte eine ganze ferne Welt vor, in der unendliche Ruhe, Milde und Feinheit herrschten. Alles sah wie ein schwaches Lächeln aus, wie eine unbestimmt schöne Erinnerung aus früher Jugend, oder wie wenn man manchmal am Morgen erwacht mit dem wohligen Gefühl, etwas sehr Schönes geträumt zu haben, ohne sich mehr erinnern zu können, was es eigentlich war. Der Hof war leer und ich, wie immer, allein und allen fremd. Aus den offenen Fenstern des Gefängnisses drangen einige polternde Laute des sonnabendlichen Scheuerns und Putzens, eine zurechtweisende Stimme wurde hie und da laut; dazwischen schlug immer wieder der Buchfink ganz hoch auf der Pappel, deren Stamm, noch ganz kahl, im schrägen Licht der scheidenden Sonne silbern glänzte. Alles atmete solchen Frieden, und meine Blicke hingen an dem mattlächelnden Wolkengebilde dort fern am Himmel, - ich stand wie gebannt im Zauber und dachte an Dich, 
an Euch alle: seht Ihr denn nicht, wie schön die Welt ist? Habt Ihr denn nicht Augen wie ich und ein Herz wie ich, um Euch zu freuen? Ich fing heute den "Wallenstein" der Ricarda Huch ${ }^{1}$ an und bin Dir herzlich dankbar für das Buch. Es erfrischt mich ungemein durch die rege Gedankenarbeit und die Freude am Schildern der menschlichen Schicksale, die so deutlich aus jeder Zeile spricht. Natürlich ist das keine exakte wissenschaftliche Arbeit; ihre Geschichtsauffassung hat gar keine ernste Basis, ist durch und durch dilettantisch und zumeist direkt schief. Aber für mich machen einen Menschen wie ein Buch nicht Ansichten, sondern der Grundstoff, aus dem Mensch und Buch bestehen. Ganz verkehrte Ansichten stören mich gar nicht, wenn ich nur innere Aufrichtigkeit, lebhafte Intelligenz und künstlerische Freude am Weltbild und Leben finde. Wie schön, dass man immer noch um die Ecke wieder Menschen entdeckt, an denen man sich freuen kann!...

Deine Nachlassbände ${ }^{2}$ will ich natürlich gern dem Julek ${ }^{3}$ zur Verfügung stellen, aber ich warte damit bis zum nächsten Besuch; denn per Post mit mir als Absender dürften sie wohl nicht so sicher in seine Hände gelangen. Was meinst Du? Übrigens kenne ich nicht einmal seine Adresse. Vielleicht kann ich sie bald Dir selbst einhändigen! Wollen wir hoffen. Lass mich sofort wissen, wenn Du Bescheid kriegst.

Meine Übersetzung ${ }^{4}$ will ich jetzt beschleuningen; ich konnte in den letzten Monaten schlecht arbeiten, nun will ich mich bessern. Hab also Geduld mit mir.

Dass Du jetzt mit Mathilde J[acob] so gut stehst (worüber auch sie mir hocherfreut berichtete) ist mir eine wahre Wohltat. Du erfährst in diesem Fall wieder, woran ich fest glaube: man kann die Menschen nur dann richtig verstehen, wenn man sie lieb hat.

Und nun sei mir heiter, hörst Du? Schimpfe nicht über das graue Wetter, studiere lieber, wie schön und mannigfaltig gerade der graue Himmel ist. Hab auch nicht so ungeduldige Eile mit dem Frühling,

1 Ihr Buch „Wallenstein. Eine Charakterstudie” erschien 1917.

${ }^{2}$ Gesammelte Schriften von Karl Marx und Friedrich Engels 185 2-1 862. Hrsg. von N. Rjasanoff. Die Úbersetzungen aus dem Englischen von Luise Kautsky. 2 Bde. Stuttgart I917.

3 Julian Marchlewski, einer der Führer der polnischen Sozialdemokratie. Unter dem Pseudonym Johannes Kämpfer hatte er an der Internationale mitgearbeitet. Am 22. Mai I916 wurde er verhaftet und nach kurzer Haft im Berliner Stadtgefängnis, der Stadtvogtei, mit Franz Mehring u.a. im Konzentrationslager Havelberg interniert. Er wurde am 22. Februar 1918 freigelassen und ging nach Russland.

4 von Wladimir Korolenkos „Die Geschichte meines Zeitgenossen”. Nachdem R. L. die Übersetzung am 28. Juli 1916 vergeblich J. $H$. W. Dietz zum Verlag angeboten hatte, erschien sie durch Vermittlung Luise Kautskys mit einer längeren Einleitung der Übersetzerin 1919 in zwei Bänden im Verlag P. Cassirer, Berlin. 
nachher geht doch alles gewöhnlich so schnell vorbei! Jetzt kann man sich wenigstens in der Erwartung freuen. Schreibe mir auch bald wieder, damit ich sehe, ob $\mathrm{Du}$ in besserer Stimmung bist. Hannes 1 berichtete mir über Euer - wie üblich - verfehltes Rendezvous an der Friedrichstrasse. Ich freue mich schon auf seine Rückkehr nach Posen.

Sei herzlich umarmt samt dem unwürdigen Igel ${ }^{2}$ von Deiner R. Grüsse von mir Bendel, ${ }^{3}$ auch Hilferding. Henriette ${ }^{4}$ könnte mir hierher mal schreiben, natürlich nicht über Politik.

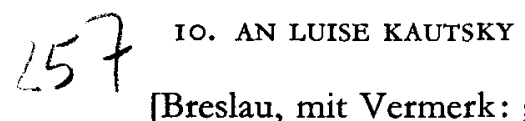

[Breslau, mit Vermerk: gel. 31.5.18 S. Oberlt.] 29. Mai I9r 8 .

Liebste, ich schrieb Dir erst gestern, muss aber Deine heute erhaltenen Zeilen gleich kurz beantworten. Was Du mir über die materielle Lage von Fritz' 5 Familie schreibst, geht mir sehr nahe, und wenn ich irgendwie helfen könnte, würde ich es sofort tun, aber Du bist über meine Möglichkeiten völlig im Irrtum. Ich bin nicht Hannes' Haupterbin, weiss auch nicht, ob es eine solche oder einen solchen überhaupt gibt. $\mathrm{H}$ [annes] hat mir nur für Lebenszeit die Zinsen von 50.000 Mark vermacht, mit dem ausdrücklichen Verbot, bis zu meinem seligen Ende über die Summe zu verfügen (vor Besorgnis, wie er schrieb, ich möchte das Geld sofort für Parteizwecke ausgeben.) Diese 50.000 Mark, über die ich keinerlei Verfügung besitze, haben sich auch noch durch Anlegung in Wertpapieren in 45.000 verwandelt, und von diesen steht mir die voraussichtliche Nutzniessung von $4 \%$ zu. Das ist alles, - wie Du siehst, kaum die Hälfte von dem, was meine Unterhaltskosten hier und meine Wohnung in Südende ausmachen. Und auch diese paar Hundert Mark soll ich wohl erst im Oktober bekommen, - soweit ich aus der Abrechnung der Stuttgarter Bank verstanden habe (über die Anlegung des Geldes etc. verfügt die

1 R.L.'s Freund Hans Diefenbach; er war als Militärarzt in Posen und verkehrte dort in der Familie von Julian Marchlewskis Bruder.

2 Hans Kautsky.

3 Karl Kautskys jüngster Sohn Benedikt.

4 Die holländische Sozialistin Henriette Roland Holst.

5 Gemeint ist Friedrich Adler, der seit dem 12 . Oktober 1917 in der Strafanstalt Stein an der Donau war. Wegen des Attentats auf den Grafen Stürgkh war er, nachdem Kaiser Karl die Bestätigung des Todesurteils abgelehnt hatte, vom Obersten Gerichtshof zu achtzehn Jahren schweren Kerkers verurteilt. Rosa Luxemburg hatte den Prozess mit stärkster Anteilnahme verfolgt. 
Schwester Hannes'). ${ }^{1}$ Damit ist wohl auch die Sache mit Zenzi ${ }^{2}$ leider erledigt, so leid es mir tut. Sonstigen Rat für Fritz' Familie wirst $\mathrm{Du}$ sicher zehnmal besser wissen, als ich hier in meiner Weltabgeschiedenheit und angeborenen Talentlosigkeit in allen Geldsachen. - Was ist mit Julek? ${ }^{3}$ Ich weiss von gar nichts, freue mich aber herzlich auf alles Gute, das ihm zugestossen sein mag. Die Mutter unserer Modekünst'erin ${ }^{4}$ grüsse von mir vielmals, sie soll ihre Gesundheit hüten. Auch den armen Hans, ${ }^{5}$ den schmachtenden Ritter in Rosen und Jasmin, grüsse ich herzlich. Wie schön muss es jetzt in meinem Südende sein, wo soviel Jasmin wächst.

Ich umarme Dich tausendmal $R$.

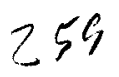

\section{I. Erklärung}

$\mathrm{Da}$ ich bereits am Mittwoch, den 25. [September], vor Beginn der Vormittagssitzung den Parteitag verlassen musste, um zur Gerichtsverhandlung nach Posen ${ }^{6}$ zu fahren, so war ich weder in der Lage, meinen Antrag betr. die Budgetabstimmung ${ }^{7}$ zu vertreten, noch bei den gegen mich in der Mittwochsitzung gerichteten Angriffen zugegen zu sein.

Zwar ist es seit jeher meine Gewohnheit, rein persönliche Angriffe nicht eines Wortes der Antwort für wert zu halten, weil für mich die Gehässigkeiten, mit denen ich von gewisser Seite hartnäckig bedacht werde und die nur ein Ausdruck politischer Gegnerschaft sind, einfach nicht in Betracht kommen.

1 Im Testament hiess es: „... Die genannte Summe soll von irgendeiner entsprechenden Instanz, z.B. von meiner Schwester, verwaltet und der Erbin der jährliche Zins bis zu ihrem Tode regelmässig ausbezahlt werden. Ich treffe diese Bestimmung, da meine ausgezeichnete Freundin in der Privatökonomie vielleicht keine ganz so geniale Meisterin ist wie in der Nationalökonomie...” L. Kautsky, „Rosa Luxemburg. Ein Gedenkbuch", Berlin I929, S. 53.

2 Sie war lange Jahre Hausgehilfin in der Familie Kautsky.

3 Julian Marchlewski war nach seiner Freilassung im Februar I91 8 nach Russland gegangen; im Juli wurde er Mitglied des Allrussischen Zentralen Exekutivkomitees.

4 Anna Nemitz; sie war in der Berliner USPD. sehr tätig und gehörte später dem Parteivorstand der SPD. am.

s Hans Kautsky.

- Es handelte sich um einen Prozess wegen Beleidigung des preussischen Kultusministers durch die 1900 in Posen veröffentlichte Broschüre "W obronie narodowości”; sie hatte datin aufgefordett, den Germanisierungsbestrebungen der preussischen Regierung energisch Widerstand zu leisten.

7 Der von ihr eingebrachte Antrag Nr. 94 wollte die sozialdemokratischen Abgeordneten in den deutschen Landtagen verpflichten, in der Gesamtabstimmung gegen das Budget zu stimmen. Protokoll des Lübecker Parteitages S. 96; der Antrag wurde zurückgezogen, ebd. S. 284 . 
Diesmal muss ich jedoch eine Ausnahme davon machen, und zwar dem Abg[eordneten] R. Fiscber gegenüber. Denn die Attaquen u.a. gegen mich, in denen sich einige Delegierte während der Pressdebatte Luft machten, gingen über meine Person hinaus und hatten den augenscheinlichen Zweck, die literarischen und politischen Qualifikationen der Richtung, die u.a. auch ich vertrete, zu verdächtigen.

Der Abg[eordnete] R. Fischer-Berlin hat nach dem VorwärtsBericht ${ }^{1}$ u.a. gesagt: „Man kann eine Auffassung vertreten in aller Schärfe, aber man darf die Meinungen der Genossen nicht fälscben und bloss in der Absicht, geistreich zu sein, zu rabulieren, zu unterschieben und zu fälschen, so zu (sic!) operieren, wie es die Genossin Luxemburg in der Millerandfrage ${ }^{2}$ und in ganz klassischer Weise in der letzten Nummer der Neuen Zeit ${ }^{3}$ getan hat."

Ich fordere hiermit den $\mathrm{Abg}$ [eordneten] R. Fischer öffentlich auf, den Wahrheitsbeweis für seine Behauptungen anzutreten und namentlich diejenigen Stellen meiner Artikel über die Millerandfrage wie über die badische Budgetabstimmung namhaft $\mathrm{zu}$ machen, wo ich "gefälscht" und „unterschoben" haben soll.

Falls er dieser Aufforderung nicht Folge leistet, werde ich mich gezwungen sehen, ihn mit dem in solchen Fällen gebräuchlichen Namen zu bezeichnen.

Berlin-Friedenau, I. Oktober I90 I.

Rosa Luxemburg.

\footnotetext{
1 Nach dem Protokoll, S. 192, lautete dieser Passus: „Man kann eine Auffassung vertreten in aller Schärfe, aber man darf die Meinungen der Genossen nicht fälschen, und bloss in der Absicht, geistreich zu erscheinen, so lange tabulisteln, bis die Wahrheit in ihrem Gegenteil erscheint. In ganz klassischer Weise hat sie das gerade wieder in der letzten Nummer der Neuten Zeit getan."

2 In fünf Aufsätzen der Neuen Zeit, Nr. 16, 17, 18, 20, 22; 16. Januar-27. Februar 1901 hatte sie "Die sozialistische Krise in Frankreich" behandelt.

${ }^{3}$ im Aufsatz "Der Parteitag und die Budgetbewilligung" in Nr. 50 vom I I. September IgOI, S. 759 ff.
} 